\title{
A128 CYTOKINE NETWORK IN THE FIRST 6 WEEKS OF RHEUMATOID ARTHRITIS ONSET
}

R Cascão, ${ }^{1}$ R A Moura, ${ }^{1}$ H Canhão, ${ }^{1,2}$ E Sousa, ${ }^{1,2}$ A F Mourão, ${ }^{1,3}$ A M Rodrigues, ${ }^{1,2}$ J Polido-Pereira, ${ }^{1,2}$ M V Queiroz, ${ }^{1,2}$ H S Rosário, ${ }^{4}$ M M Souto-Carneiro, ${ }^{5}$ L Graça, ${ }^{6}$ J E Fonseca1,2 ${ }^{1}$ Rheumatology Research Unit, Instituto de Medicina Molecular, Faculdade de Medicina da Universidade de Lisboa, Lisboa, Portugal; ${ }^{2}$ Rheumatology Department, Hospital de Santa Maria, Lisboa, Portugal; ${ }^{3}$ Rheumatology Department, Hospital Egas Moniz, Lisboa, Portugal; ${ }^{4}$ Microvascular Biology and Inflammation Unit, Instituto de Medicina Molecular, Faculdade de Medicina da Universidade de Lisboa, Lisboa, Portugal; ${ }^{5}$ Center for Neurosciences and Cell Biology, Autoimmunity Group, Coimbra, Portugal; ${ }^{6}$ Cellular Immunology Unit, Instituto de Medicina Molecular, Faculdade de Medicina da Universidade de Lisboa, Lisboa, Portugal

10.1136/ard.2010.129643e

Introduction Rheumatoid arthritis (RA) is a chronic inflammatory disease mainly characterised by synovial hyperplasia and joint destruction. Although the aetiopathology of this autoimmune disease is not completely understood, it is known that it is associated with a misregulation of both the cellular immune system and the cytokine network. Nevertheless, little is known about the blood cytokine milieu in the first few weeks of RA.

Objectives To determine concentration of cytokines in serum samples from patients with undiagnosed polyarthritis of $<6$ weeks' duration that later evolved into RA (very early RA, VERA) or into other conditions (non-RA, VEA).

Methods: Interleukin (IL)1 $\beta$, IL2, IL4, IL6, IL8, IL10, IL12 (p70), IL17A, IL18, IL22, IL23, interferon (IFN) $\gamma$, leptin, MCP-1, MIP-1 $\alpha$, OPG, transforming growth factor $\beta$ (TGF $\beta$ ) and tumour necrosis factor (TNF) were measured in serum samples of patients and controls by FlowCytomix assay kit. IL21, APRIL and BAFF levels were determined by ELISA.

Results Patients with VERA have increased levels of IL1 $\beta$, IL6, IL8, IL17A, IL22, APRIL, BAFF, MCP-1 and leptin at baseline in comparison with healthy controls. IL1 $\beta$, IL8, IL22, leptin and BAFF were higher in patients with VERA than in those with VEA. Both patients with VERA and those with VEA have reduced levels of TGF $\beta$ compared with controls. No statistically significant differences could be observed for IL4, IL12, IL18, MIP-1 $\alpha$ or OPG. IL2, IL10, IL23, IFN $\gamma$ and TNF were undetectable or very low in all patients with early arthritis.

Conclusion In very early arthritis the increased production of serum IL1 $\beta$, IL8, IL17A and MCP-1 could support the migration of neutrophils and macrophages towards the synovium. The increased levels of IL6, APRIL and BAFF generated during the inflammatory process could activate B cells towards the synovial membrane. Furthermore, the increase in IL1 $\beta$ and IL6 might be related to the early driving of Th17 differentiation observed in these patients. These observations might be of interest for targeting selection of patients with very early arthritis.

$\mathrm{RC}$ and RAM contributed equally to this work. 